\title{
Osteomyelitis Due to Mycobacterium goodii in an Adolescent, United States
}

\author{
Alejandro Diaz, Monica I. Ardura, Huanyu Wang, \\ Stella Antonara, Christopher P. Ouellette
}

Author affiliations: Nationwide Children's Hospital and The Ohio State University, Columbus, Ohio, USA (A. Diaz, M.I. Ardura, H. Wang, C.P. Ouellette); Riverside Methodist Hospital, Columbus (S. Antonara)

\section{DOI: https://doi.org/10.3201/eid2611.200206}

Osteomyelitis is a rare clinical manifestation of infection with nontuberculous mycobacteria (NTM). We report an adolescent with femoral osteomyelitis associated with prosthetic material due to an emerging pathogen, Mycobacterium goodii. Application of secA1 and $16 \mathrm{~S}$ ribosomal RNA gene sequencing reliably determined the NTM species, enabling targeted antimicrobial therapy.

$\mathrm{N}$ ontuberculous mycobacteria (NTM) are an emerging cause of human infections, likely because of improved detection methods and an increasing high-risk population (1-3). Conventional methods to identify NTM species rely on phenotypic characteristics to differentiate the most common species, but these labor-intensive and time-consuming methods delay final identification and appropriate therapy (2). Sequencing of 16S rRNA and secA1 (essential secretory protein SecA1) genes provides an accurate and cost-effective method for NTM identification, offering a turnaround time of 1-2 days compared with 2-6 weeks for results from conventional methods (4).

$M$. goodii is a rapidly growing mycobacterium that can be nonpigmented or late-pigmented. Before 1999 , the original classification of the 3 species in the M. smegmatis group identified 28 isolates of M. goodii, which most often were associated with posttraumatic wound infections (5). Since then, $M$. goodii has been implicated in infections related to prosthetic devices and penetrating trauma. Three recent reports detail 19 cases of $M$. goodii infections in patients with a mean age of 60 years (range 6-85 years). Types of infection included prosthetic device or pocket infection $(n=12)$, wound infection $(n=3)$, endocarditis $(n=1)$, pneumonia $(n=$ $2)$, and endophthalmitis $(n=1)(6-8)$. We noted only 3 pediatric cases in the literature: 2 cases of pneumonia, 1 in a 15-year-old girl and 1 in a 7-week-old infant; and 1 soft tissue infection in a 6-year-old boy $(6,9,10)$.

We report a 15-year-old male patient with severe bilateral knee flexion contractures who underwent bilateral femoral extension osteotomies with hardware implantation. Two months later, he had intermittent low-grade fevers, right thigh pain, and surgical wound dehiscence with discharge. Initial laboratory results showed elevated leukocyte count, erythrocyte sedimentation rate, and C-reactive protein (Figure). An incision and drainage was performed but the femoral hardware was retained; 4 days later, a second incision and drainage was performed with primary closure.

Acid-fast bacillus (AFB) cultures were obtained; after 22 days, NTM growth was identified. Empiric therapy was initiated with intravenous (IV) amikacin $(15 \mathrm{mg} / \mathrm{kg} 1 \times / \mathrm{d})$, IV cefoxitin (3,000 mg every $8 \mathrm{~h})$, oral azithromycin $(250 \mathrm{mg} 1 \times / \mathrm{d})$, and oral ciprofloxacin (500 mg every $12 \mathrm{~h}$ ). Two weeks later, the patient underwent a third incision and drainage and hardware was removed because of recrudescent fever and surgical site discharge. AFB tissue cultures from bone again grew NTM. Sequencing of the secA1 and $16 \mathrm{~S}$ genes from all NTM isolates identified $M$. goodii (Appendix Figure, https://wwwnc.cdc.gov/ EID/article/26/11/20-0206-App1.pdf). The patient's therapy was modified to oral trimethoprim/sulfamethoxazole (TMP/SMX; $320 \mathrm{mg} \mathrm{2 \times /d,6} \mathrm{mg/kg/}$ dose based on TMP component), with continued IV amikacin and oral ciprofloxacin. Antimicrobial susceptibility testing results confirmed susceptibility to TMP/SMX, ciprofloxacin, amikacin, and doxycycline but noted resistance to clarithromycin and cefoxitin. Amikacin was discontinued after a total of 36 days of therapy.

Five months after his last surgical intervention, the patient had clear discharge from his right thigh. A small superficial skin abscess was noted on magnetic resonance imaging. Fine needle aspiration of the fluid collection was performed from which AFB cultures were sterile but universal bacterial $16 \mathrm{~S}$ rDNA PCR detected M. goodii. Given the potential for antimicrobial resistance, oral doxycycline $(100 \mathrm{mg} 2 \times / \mathrm{d})$ was added to the patient's antimicrobial drug regimen. The 3-drug regimen was continued for an additional 4 months. Repeat imaging at the end of therapy showed no evidence of fluid reaccumulation, and the patient has not had an infection relapse for 10 months after discontinuation of antimicrobial drug therapy. No other M. goodii infections have been identified at our institution since this case.

NTM osteomyelitis treatment can be challenging. Management strategies include prolonged antimicrobial drug therapy, surgical debridement, and removal of foreign material (2). M. goodii usually is susceptible to TMP/SMX, amikacin, ciprofloxacin, 


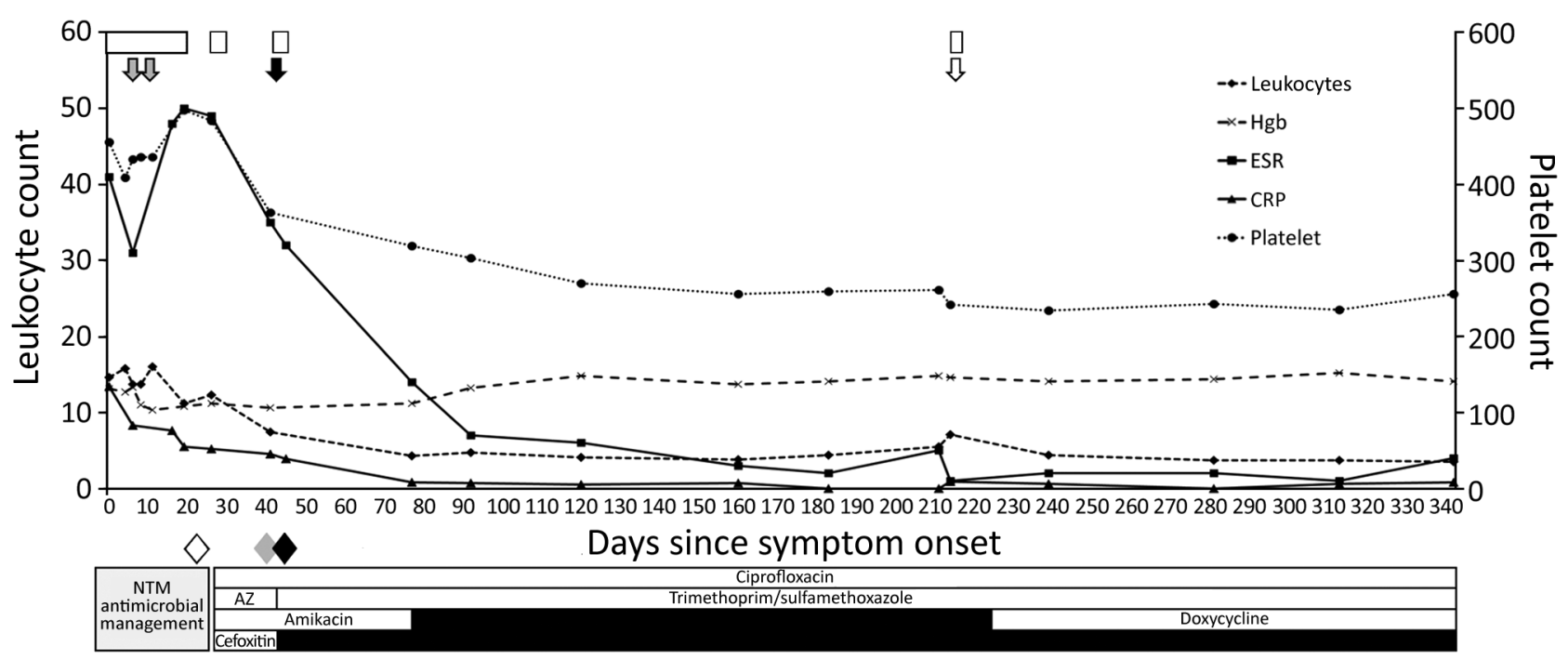

Figure. Timeline of laboratory values, surgical interventions, notification of pertinent culture results, and antimicrobial drug therapy in a case of osteomyelitis due to Mycobacterium goodii in an adolescent, United States. White boxes represent periods of hospitalization. Gray arrows indicate dates of surgical intervention with cultures obtained and femoral hardware retained. Solid black arrow indicates surgical intervention with cultures obtained and femoral hardware removed. Shaded arrow indicates interventional radiology aspiration of subcutaneous fluid collection with cultures obtained. Shaded diamond indicates first notification of nontuberculous mycobacterium growth. Solid gray diamond indicates first notification of $M$. goodii by $16 S$ and secA1 sequencing. Solid black diamond indicates first notification of susceptibility results for $M$. goodii. AZ, azithromycin; CRP, C-reactive protein; ESR, erythrocyte sedimentation rate; Hgb, hemoglobin; NTM, nontuberculous mycobacteria.

imipenem, and doxycycline (5). However, M. goodii is intrinsically resistant to macrolides and rifampin, which commonly are used for empirical therapy of NTM infections; early species identification is crucial to ensuring effective and timely treatment $(2,5,6)$. Optimal treatment is unknown, but a combination of $\geq 2$ active drugs, for a minimum of 6 months, combined with surgical debridement and hardware removal, is recommended to ensure clinical and bacteriological cure and prevent antimicrobial resistance $(2,6)$.

Our case shows similarities to prior adult reports, specifically prosthetic-associated M. goodii infection, and further highlights the emergence of this pathogen in the pediatric population. Given the repeated culture-positive results from our patient, we do not believe this case was the result of an environmental contaminant. In addition, no other cases of $M$. goodii infection have been identified at our institution to suggest nosocomial infection, but we cannot definitively exclude this mode of acquisition.

In conclusion, our case highlights $M$. goodii as an emerging pediatric NTM pathogen. These findings underscore the use of secA1 and 16S rRNA sequencing for rapid species identification to enable timely and effective antimicrobial drug therapy.

\section{About the Author}

Dr. Diaz is a pediatric infectious disease fellow at Nationwide Children's Hospital, Columbus, Ohio, USA. His research interests include antibiotic stewardship and antimicrobial resistance.

\section{References}

1. Brown-Elliott BA, Wallace RJ Jr. Clinical and taxonomic status of pathogenic nonpigmented or late-pigmenting rapidly growing mycobacteria. Clin Microbiol Rev. 2002;15:71646. https:// doi.org/10.1128/CMR.15.4.716-746.2002

2. Griffith DE, Aksamit T, Brown-Elliott BA, Catanzaro A, Daley C, Gordin F, et al.; ATS Mycobacterial Diseases Subcommittee; American Thoracic Society; Infectious Disease Society of America. An official ATS/IDSA statement: diagnosis, treatment, and prevention of nontuberculous mycobacterial diseases. Am J Respir Crit Care Med. 2007;175:367-416. https://doi.org/10.1164/rccm.200604571ST

3. Tebruegge M, Pantazidou A, MacGregor D, Gonis G, Leslie D, Sedda L, et al. Nontuberculous Mycobacterial Disease in Children - Epidemiology, Diagnosis \& Management at a Tertiary Center. PLoS One. 2016; 11:e0147513. https:/ / doi.org/10.1371/journal.pone.0147513

4. Cook VJ, Turenne CY, Wolfe J, Pauls R, Kabani A. Conventional methods versus $16 \mathrm{~S}$ ribosomal DNA sequencing for identification of nontuberculous mycobacteria: cost analysis. J Clin Microbiol. 2003;41:1010-5. https:// doi.org/10.1128/JCM.41.3.1010-1015.2003

5. Brown BA, Springer B, Steingrube VA, Wilson RW, Pfyffer GE, Garcia MJ, et al. Mycobacterium wolinskyi sp. nov. 
and Mycobacterium goodii sp. nov., two new rapidly growing species related to Mycobacterium smegmatis and associated with human wound infections: a cooperative study from the International Working Group on Mycobacterial Taxonomy. Int J Syst Bacteriol. 1999;49:1493-511. https:/ / doi.org/10.1099/00207713-49-4-1493

6. Salas NM, Klein N. Mycobacterium goodii: an emerging nosocomial pathogen: a case report and review of the literature. Infect Dis Clin Pract (Baltim Md). 2017;25:62-5. https://doi.org/10.1097/IPC.0000000000000428

7. Harper G, Ong JL, Costanigro L. Oh Goody! Two additional Mycobacterium goodii infections. Open Forum Infectious Diseases. 2015;2(suppl_1):576. https://doi.org/10.1093/ ofid/ofv133.450

8. Parikh RB, Grant M. Mycobacterium goodii endocarditis following mitral valve ring annuloplasty. Ann Clin Microbiol Antimicrob. 2017;16:14. https:/ / doi.org/10.1186/s12941-0170190-4

9. Goussard P, Rabie H, Morrison J, Schubert PT. Superinfection with Mycobacteria goodii in a young infant with exogenous lipoid pneumonia. Pediatr Pulmonol. 2019;54:1345-7. https://doi.org/10.1002/ppul.24355

10. Hougas JE III, Bruneteau RJ, Varman M. Mycobacterium goodii infection of skin graft in an immunocompetent child. Infect Dis Clin Pract. 2011;19:146-7. https:/ / doi.org/10.1097/ IPC.0b013e3182002df1

Address for correspondence: Christopher Ouellette, Division of Infectious Diseases and Host Defense Program, Nationwide Children's Hospital, 700 Children's Dr, Rm C5C-J5432, Columbus, OH 43205, USA; email: christopher.ouellette@ nationwidechildrens.org

\section{Sporotrichosis Cases in Commercial Insurance Data, United States, 2012-2018}

\author{
Kaitlin Benedict, Brendan R. Jackson \\ Author affiliation: Centers for Disease Control and Prevention, \\ Atlanta, Georgia, USA
}

DOI: https://doi.org/10.3201/eid2611.201693

The geographic distribution of sporotrichosis in the United States is largely unknown. In a large commercial health insurance database, sporotrichosis was rare but most frequently occurred in southern and south-central states. Knowledge about where sporotrichosis is most likely to occur is essential for increasing clinician awareness of this rare fungal disease.
Sporotrichosis is an infection caused by the fungus Sporothrix. The infection typically follows cutaneous inoculation and involves the skin, subcutaneous tissue, and lymph nodes; pulmonary or disseminated disease occurs less frequently and usually affects immunocompromised persons (1). Sporothrix exists nearly worldwide in soil and decaying plant matter, but many unanswered questions remain about its precise ecologic niche (2). In the United States, its geographic distribution is poorly understood. Knowledge about where sporotrichosis is most likely to occur can help healthcare providers recognize and treat it earlier and help public health officials focus prevention messages.

We used the MarketScan Research Databases (IBM, https://www.ibm.com) to examine the geographic distribution of sporotrichosis in the United States. These databases comprise health insurance claims data from outpatient visits, prescriptions, and hospitalizations for employees, dependents, and retirees throughout the United States. In 2018, the databases contained records for $\approx 27$ million persons. MarketScan data are fully de-identified; thus, the Centers for Disease Control and Prevention institutional review board did not need to approve this study.

To query the database, we used Treatment Pathways (IBM), a web-based platform, that comprises data from persons with health insurance plans that contributed prescription drug information to the MarketScan databases. We used data from February 1, 2012-December 31, 2018, to identify sporotrichosis patients using code 117.1 from the International Classification of Diseases (ICD), Ninth Revision, Clinical Modification and code B42 from the ICD, 10th Revision, Clinical Modification (ICD-10-CM). We used the primary beneficiary's state of residence to calculate average annual state-specific rates per 1 million MarketScan enrollees. We evaluated underlying conditions on or in the month before sporotrichosis diagnosis, demographic features, and type of sporotrichosis.

Of $\approx 76$ million unique patients during 2012-2018, 1,322 had a sporotrichosis diagnosis code. For 1,236 $(93.5 \%)$ of those, information was available about state of residence. The average annual rate of sporotrichosis cases per 1 million enrollees was highest in Oklahoma (6.1), Michigan (3.9), Kansas (3.5), and Kentucky (3.5) (Figure). Nationwide, the average annual rate was 2.0 cases/1 million enrollees.

For the 1,252 patients continuously enrolled during the month before their diagnosis, median age was 54 years; most (62\%) patients were female (Table). The most common underlying conditions we evaluated were diabetes $(7 \%)$, immune-mediated inflammatory disease $(3 \%)$, and chronic obstructive pulmonary 